Otterbein University

Digital Commons @ Otterbein

Physics Faculty Scholarship

Physics

3-8-1999

\title{
Renormalized Effective QCD Hamiltonian: Gluonic Sector
}

David G. Robertson

Otterbein University

E.S. Swanson

North Carolina State U. \& Jefferson Lab

A.P. Szczepaniak

Indiana University

C.R. Ji

S.R. Cotanch

North Carolina State University

Follow this and additional works at: https://digitalcommons.otterbein.edu/phys_fac

Part of the Physics Commons

\section{Repository Citation}

Robertson, David G.; Swanson, E.S.; Szczepaniak, A.P.; Ji, C.R.; and Cotanch, S.R., "Renormalized Effective QCD Hamiltonian: Gluonic Sector" (1999). Physics Faculty Scholarship. 16.

https://digitalcommons.otterbein.edu/phys_fac/16

This Article is brought to you for free and open access by the Physics at Digital Commons @ Otterbein. It has been accepted for inclusion in Physics Faculty Scholarship by an authorized administrator of Digital Commons @ Otterbein. For more information, please contact digitalcommons07@otterbein.edu. 


\title{
Renormalized effective QCD Hamiltonian: Gluonic sector
}

\author{
D. G. Robertson, ${ }^{1}$ E. S. Swanson, ${ }^{1,2}$ A. P. Szczepaniak, ${ }^{3}$ Chueng-Ryong Ji, ${ }^{1}$ and S. R. Cotanch ${ }^{1}$ \\ ${ }^{1}$ Department of Physics, North Carolina State University, Raleigh, North Carolina 27695-8202 \\ ${ }^{2}$ Jefferson Laboratory, 12000 Jefferson Ave., Newport News, Virginia, 23606 \\ ${ }^{3}$ Department of Physics and Nuclear Theory Center, Indiana University, Bloomington, Indiana 47405
}

(Received 26 October 1998; published 8 March 1999)

\begin{abstract}
Extending previous QCD Hamiltonian studies, we present a new renormalization procedure which generates an effective Hamiltonian for the gluon sector. The formulation is in the Coulomb gauge where the QCD Hamiltonian is renormalizable and the Gribov problem can be resolved. We utilize elements of the Głazek and Wilson regularization method but now introduce a continuous cut-off procedure which eliminates non-local counterterms. The effective Hamiltonian is then derived to second order in the strong coupling constant. The resulting renormalized Hamiltonian provides a realistic starting point for approximate many-body calculations of hadronic properties for systems with explicit gluon degrees of freedom. [S0556-2821(99)01807-X]

PACS number(s): 12.39.Mk, 11.10.Ef, 11.10.Gh, 12.39.Ki
\end{abstract}

\section{INTRODUCTION}

A quantum field theory that is local will ensure that causality is not violated; however, application of such a theory in four-dimensional space-time will generate divergences. About 50 years ago in a series of papers [1], Dyson developed renormalization to specifically address such infinities in QED. He ingeniously converted the divergence problem into a useful criterion for selecting appropriate theories, namely that they should be renormalizable. Today the view towards renormalization is somewhat more practical and perhaps limited in scope. Guided by Wilson and Weinberg, theories are now regarded more as partial descriptions rather than complete constructs. Accordingly, contemporary approaches embody the concept of an effective theory that only includes the necessary degrees of freedom appropriate to a specific energy range.

In this paper, we follow this philosophy and consider the development of an effective QCD Hamiltonian for systems with explicit gluon excitations in the few $\mathrm{GeV}$ energy range. This work extends our previous Hamiltonian glueball investigation [2] and recent renormalization treatment of the quark sector [3]. Our motivation is three-fold. First, we seek a rigorous formalism connecting QCD to effective and tractable model Hamiltonians. Second, we wish to develop a realistic theoretical treatment to accurately describe hadron structure, especially systems with gluonic degrees of freedom such as glueballs and hybrids. Finally, we want to understand the basic confinement and chiral symmetry breaking mechanisms.

We begin with the exact QCD Hamiltonian formulated in the Coulomb gauge and focus upon the pure gluonic sector. Renormalizing the quark Hamiltonian, which has been previously studied using a sharp cut-off regularization [3], and the quark-glue sector will be addressed in a future publication. There are several reasons for utilizing the Coulomb gauge in which the divergence of the color vector potential vanishes $(\nabla \cdot \mathbf{A}=0)$. As detailed by Zwanziger [4], not only is the Hamiltonian renormalizable in this gauge but the Gribov problem [5] can also be resolved. The essence of the Gribov problem is that specification of $\nabla \cdot \mathbf{A}=0$ does not uniquely fix the gauge in non-Abelian gauge theories. In general, there are many copies of each gauge field configuration, all with the same divergence, that are related by gauge transformations. As the true physical configuration space of a gauge theory is the set of gauge potentials modulo local gauge transformations, one must select a single representative from each set of gauge-equivalent configurations. The resulting sub-set, $\mathcal{A}_{\text {phys }}$, of independent field configurations is known as the fundamental modular region.

A convenient characterization of $\mathcal{A}_{\text {phys }}$ is given by the "minimal" Coulomb gauge, obtained by minimizing a suitably-chosen functional along gauge orbits. We define the $L_{2}$ norm of a field configuration along a gauge orbit by

$$
F_{\mathbf{A}}[G]=\operatorname{Tr} \int d^{3} x\left(\mathbf{A}^{G}\right)^{2},
$$

where $G(\mathbf{x})$ is a gauge transformation and

$$
\mathbf{A}^{G}=G \mathbf{A} G^{\dagger}-G \nabla G^{\dagger} .
$$

Then $\mathcal{A}_{\text {phys }}$ is specified by choosing from each gauge orbit the configuration which globally minimizes $F$. It is straightforward to verify that the extrema of $F$ satisfy $\nabla \cdot \mathbf{A}=0$, so that this defines a Coulomb gauge. Furthermore, at a minimum of $F$ the Faddeev-Popov operator satisfies $M$ $\equiv-\nabla \cdot \mathbf{D} \geqslant 0$ (i.e., its eigenvalues are non-negative). Here $\mathbf{D}=\nabla-i g \mathbf{A}$ is the covariant derivative. One can show that configurations for which $M=0$ occur only on the boundary of $\mathcal{A}_{\text {phys }}$. These actually represent gauge copies and must be identified to give the full physical configuration space. Needless to say, the resulting functional space has an extremely complicated structure. Zwanziger has shown how to rigorously implement the restriction to $\mathcal{A}_{\text {phys }}$ in the infinitevolume limit for a gauge-fixed version of the lattice QCD Hamiltonian formulation [4].

The confinement phenomenon in QCD has two complementary aspects: (1) there is a long range attractive potential between colored sources; (2) the gluons which mediate this force are absent from the spectrum of physical states. This poses something of a mystery in a covariant gauge, however, 
since for small $q^{2}$, (1) suggests that the gluon propagator should be more singular than $1 / q^{2}$, while (2) implies the propagator is suppressed. Thus the mechanism for confinement is not particularly transparent [6].

In the Coulomb gauge, in contrast, these two aspects can comfortably co-exist: the long range force is represented by the instantaneous Coulomb interaction, while the (transverse) gluon propagator can simultaneously be suppressed at $q^{2}$ $=0$. Indeed, a detailed analysis suggests precisely this picture. The key issue is the proper identification of the fundamental modular region. Zwanziger has argued recently that restricting the configuration space to the fundamental region results in the Coulomb term acquiring a singular contribution at long distances [4]. ${ }^{1}$ This singularity is also connected to a suppression of the propagator for the would-be-physical transverse gluons at $q^{2}=0$. This suggests a rather appealing scenario for confinement in the Coulomb gauge.

For these reasons and because we are interested in understanding confinement, we adopt the Coulomb gauge and only work with transverse components of the gluon fields. Next we divide the Hamiltonian $H$ into a free part, $H_{0}$, which is the full Hamiltonian evaluated with zero coupling constant, and interaction defined by $H_{I}=H-H_{0}$. We work in a Fock space spanned by eigenfunctions of $H_{0}$ with eigenvalues $E_{n}$. In general matrix elements of the interaction diverge in this space and we regulate by suppressing contributions between states in which the energy difference $\left|E_{n}-E_{m}\right|$ is larger than scale set by a cut-off parameter $\Lambda$. However, we do not use the sharp cut-off procedure of Głazek and Wilson [7] since this generates unacceptable non-local interactions upon renormalization. These may be avoided by using a smooth regulator. This interesting result is not attributed to the specific Hamiltonian or choice of gauge since this feature emerges in scalar field theories as well. Although our theory is now rendered finite it is inappropriately dependent upon the parameter $\Lambda$. Furthermore, since this parameter is governed by free energies, the theory is no longer Lorentz invariant. It is also not gauge invariant but after renormalizing both symmetries will be restored along with elimination of all the cut-off dependence. In addition to this regularization we also suppress divergent matrix elements of one-body operators which arise from normal ordering the two-body interaction. Again a continuous, exponential cut-off regulator is used and cut-off sensitivity is removed by renormalization.

Renormalization is achieved by adding a counterterm Hamiltonian which can be expressed in terms of unknown coefficients and a complete set of local operators that respect the symmetries of the regularized Hamiltonian. An effective and elegant means for determining these coefficients is by performing a similarity transformation [7]. Because the transformation is unitary, the physical content is preserved but the cut-off is now reduced to a lower value $\Lambda_{1}$. The transformation also introduces new interactions which incorporate the physics contained between the scales $\Lambda$ and $\Lambda_{1}$. A

\footnotetext{
${ }^{1}$ This mechanism for confinement was first suggested by Gribov [5].
}

key aspect of the renormalization scheme is the requirement that the transformed Hamiltonian be form invariant, i.e. it maintains its mathematical structure but with $\Lambda_{1}$ now replacing $\Lambda[8]$.

Because $\Lambda$ can be arbitrarily large, we select an initial scale which is amenable to perturbation theory. The regularized Hamiltonian is expanded in powers of the coupling constant $g$ and for this study only terms to order $g^{2}$ are retained. While the similarity transformation rigorously evolves the scale it cannot account for confinement at hadronic energies. This constitutes our main theoretical omission. For hadronic applications confinement may be described by supplementing the renormalized Hamiltonian with a confining interaction. We will address this issue further in a future publication.

At this point we have obtained an effective Hamiltonian suitable for application to the vacuum and excited gluonic states, i.e. glueballs. However, our experience [2,9] indicates that an improved description can be obtained by performing an additional similarity transformation to a quasiparticle basis. This is a BCS (Bogoliubov) rotation which mixes the bare parton gluon creation and annihilation operators. As previously demonstrated [2,9] through variational calculations for an unrenormalized Hamiltonian in both the quark and gluon sectors, dynamical chiral symmetry breaking occurs and reasonable values for condensates and constituent masses are obtained. Similar vacuum BCS variational calculations using our renormalized effective gluon Hamiltonian are in progress and will be reported in a future publication along with many-body Tamm-Dancoff and random phase approximations for the excited hadron (glueball) spectrum.

This paper is divided into five sections and one appendix. In the next section our notation is established and the canonical QCD Hamiltonian is specified. Section III addresses our regularization scheme. Section IV presents the main result of the paper. It includes a discussion of the counterterm Hamiltonian and the form invariance renormalization scheme. This is followed by a sub-section devoted to the similarity transformation which yields the final renormalized effective interaction. A detailed representation of the canonical Hamiltonian in Fock space is presented in the Appendix.

\section{CANONICAL HAMILTONIAN}

Our starting point is the canonical QCD Hamiltonian in the Coulomb gauge $\nabla \cdot \mathbf{A}=0$ [10]. We denote spatial vectors by bold-faced quantities and use the matrix notation for the gauge fields: $\mathbf{A} \equiv \mathbf{A}^{a} T^{a}$, with $T^{a}$ the generators of $\mathrm{SU}\left(N_{c}\right)$ in the fundamental representation, satisfying $\left[T^{a}, T^{b}\right]$ $=i f^{a b c} T^{c}$. The gauge covariant derivative is $\mathbf{D}=\nabla-i g \mathbf{A}$ operating on objects in the fundamental representation and $\mathbf{D}=\nabla-i g[\mathbf{A}, \quad]$ for objects in the adjoint representation.

The dynamical degrees of freedom are the transverse gauge fields $\mathbf{A}$, their conjugate momenta $\boldsymbol{\Pi}$ (also transverse) and the quark field $\psi$. The canonical Hamiltonian takes the form

$$
H_{\mathrm{can}}=H_{q}+H_{g}+H_{C}+H_{q g}
$$


with

$$
\begin{gathered}
H_{q}=\int d^{3} x \psi^{\dagger}(\mathbf{x})[-i \alpha \cdot \nabla+\beta m] \psi(\mathbf{x}) \\
H_{g}=\operatorname{Tr} \int d^{3} x\left[\mathcal{J}^{-1} \mathbf{\Pi} \cdot \mathcal{J} \mathbf{\Pi}+\mathbf{B} \cdot \mathbf{B}\right] \\
H_{C}=\frac{1}{2} g^{2} \int d^{3} x d^{3} y \mathcal{J}^{-1} \rho^{a}(\mathbf{x}) K^{a b}(\mathbf{x}, \mathbf{y}) \mathcal{J} \rho^{b}(\mathbf{y}) \\
H_{q g}=-g \int d^{3} x \psi^{\dagger}(\mathbf{x}) \alpha \cdot \mathbf{A}(\mathbf{x}) \psi(\mathbf{x}) .
\end{gathered}
$$

Here $\alpha^{i} \equiv \gamma^{0} \gamma^{i}$ and $\beta \equiv \gamma^{0}$ are the Dirac matrices, $\mathcal{J}$ is the Faddeev-Popov determinant,

$$
\mathcal{J}=\operatorname{det}[\nabla \cdot \mathbf{D}],
$$

normalized so that $\operatorname{det}\left[\nabla^{2}\right]=1$, and $\mathbf{B}$ is the non-Abelian magnetic field with components

$$
B_{i}^{a}=\epsilon_{i j k} \nabla_{j} A_{k}^{a}+\frac{g}{2} \epsilon_{i j k} f^{a b c} A_{j}^{b} A_{k}^{c} .
$$

In the Coulomb term, Eq. (2.4), the kernel $K$ is represented in "matrix" notation as

$$
K^{a b}(\mathbf{x}, \mathbf{y})=\left\langle\mathbf{x}, a\left|(\nabla \cdot \mathbf{D})^{-1}\left(-\nabla^{2}\right)(\nabla \cdot \mathbf{D})^{-1}\right| \mathbf{y}, b\right\rangle
$$

and the color charge density $\rho^{a}$ includes both quark and gluonic contributions

$$
\rho^{a}(\mathbf{x})=\psi^{\dagger}(\mathbf{x}) T^{a} \psi(\mathbf{x})+f^{a b c} \mathbf{A}^{b}(\mathbf{x}) \cdot \Pi^{c}(\mathbf{x}) .
$$

The nonvanishing canonical commutator for the gauge field is

$$
\left[A_{i}^{a}(\mathbf{x}), \Pi_{j}^{b}(\mathbf{y})\right]=i \delta^{a b}\left(\delta_{i j}-\frac{\nabla_{i} \nabla_{j}}{\nabla^{2}}\right) \delta^{(3)}(\mathbf{x}-\mathbf{y}) .
$$

The Fourier decompositions are

$A_{i}^{a}(\mathbf{x})=\int \frac{d^{3} k}{(2 \pi)^{3}} \frac{1}{\sqrt{2 \omega_{\mathbf{k}}}}\left[a_{i}^{a}(\mathbf{k})+a_{i}^{a \dagger}(-\mathbf{k})\right] e^{i \mathbf{k} \cdot \mathbf{x}}$

$\Pi_{i}^{a}(\mathbf{x})=-i \int \frac{d^{3} k}{(2 \pi)^{3}} \sqrt{\frac{\omega_{\mathbf{k}}}{2}}\left[a_{i}^{a}(\mathbf{k})-a_{i}^{a \dagger}(-\mathbf{k})\right] e^{i \mathbf{k} \cdot \mathbf{x}}$,

with $\omega_{\mathbf{k}}=|\mathbf{k}| \equiv k$. In momentum space Eq. (2.10) reduces to

$\left[a_{i}^{a}(\mathbf{k}), a_{j}^{b \dagger}\left(\mathbf{k}^{\prime}\right)\right]=\delta^{a b}(2 \pi)^{3} \delta^{(3)}\left(\mathbf{k}-\mathbf{k}^{\prime}\right) D_{i j}(\mathbf{k})$,

where

$$
D_{i j}(\mathbf{k}) \equiv \delta_{i j}-\frac{k_{i} k_{j}}{\mathbf{k}^{2}} .
$$

Note that transversality of $\mathbf{A}$ and $\boldsymbol{\Pi}$ requires

$$
\mathbf{k} \cdot \mathbf{a}^{a}(\mathbf{k})=\mathbf{k} \cdot \mathbf{a}^{a \dagger}(\mathbf{k})=0 .
$$

These operators can also be decomposed in terms of orthogonal polarization vectors

$$
a_{i}^{a}(\mathbf{k})=\sum_{\lambda=1,2} \epsilon_{i}(\mathbf{k}, \lambda) a^{a}(\mathbf{k}, \lambda) .
$$

For the Fermi field the operator expansion takes the form

$$
\begin{aligned}
\psi(\mathbf{x})= & \sum_{s} \int \frac{d^{3} k}{(2 \pi)^{3}}[u(\mathbf{k}, s) b(\mathbf{k}, s) \\
& \left.+v(-\mathbf{k}, s) d^{\dagger}(-\mathbf{k}, s)\right] e^{i \mathbf{k} \cdot \mathbf{x}},
\end{aligned}
$$

where $s$ labels the helicity (color and flavor indices have been suppressed) and a nonrelativistic normalization is used such that $u^{\dagger} u=v^{\dagger} v=1$. The nonvanishing canonical anticommutators are then

$$
\begin{aligned}
\left\{b(\mathbf{k}, s), b^{\dagger}\left(\mathbf{k}^{\prime}, s^{\prime}\right)\right\} & =\left\{d(\mathbf{k}, s), d^{\dagger}\left(\mathbf{k}^{\prime}, s^{\prime}\right)\right\} \\
& =\delta_{s s^{\prime}}(2 \pi)^{3} \delta^{(3)}\left(\mathbf{k}-\mathbf{k}^{\prime}\right) .
\end{aligned}
$$

It is straightforward to express the canonical Hamiltonian in the Fock representation. The result to $\mathcal{O}\left(g^{2}\right)$ is given in the Appendix.

\section{REGULARIZATION SCHEME}

Perturbative schemes for renormalizing Hamiltonians typically suffer from technical problems related to the occurrence of vanishing energy denominators. An elegant way of avoiding this difficulty is the cut-off method of Głazek and Wilson [7]. This approach uses the basis formed by eigenstates of the free Hamiltonian $H_{0}$, with eigenvalues $E_{n}$. The theory is then regulated by suppressing matrix elements of $H$ between states for which the difference in free energies $E_{n m} \equiv E_{n}-E_{m}$ is large. Specifically, matrix elements of the regulated Hamiltonian $H(\Lambda)$ are defined by

$$
\langle n|H(\Lambda)| m\rangle \equiv E_{n} \delta_{n m}+f_{n m}(\Lambda)\left\langle n\left|H_{I}\right| m\right\rangle,
$$

where $H_{I}=H-H_{0}$ is the interaction. Here $f_{n m}(\Lambda)$ is some convenient function which approaches unity for $\left|E_{n m}\right| \ll \Lambda$ and vanishes for $\left|E_{n m}\right| \gg \Lambda$. In this work we employ a smooth cutoff of the form ${ }^{2}$

$$
f_{n m}(\Lambda)=e^{-E_{n m}^{2} / \Lambda^{2}} .
$$

Note that diagonal matrix elements are unmodified.

A few remarks on this regularization scheme are in order. First, it does not reduce the size of the Hilbert space, that is,

\footnotetext{
${ }^{2}$ We have also investigated a sharp cut-off, with $f_{n m}=\theta(\Lambda$ $\left.-\left|E_{n m}\right|\right)$. However, this choice leads to pathologies in the renormalized Hamiltonian, specifically nonlocal counterterms. These terms also arise in scalar field theory and thus have nothing to do with the choice of the Coulomb gauge. This issue is discussed further below.
} 
no states are removed from the theory. Reduction to a finite number of degrees of freedom-which is necessary for the nonperturbative calculations we wish eventually to perform-occurs later, after the variational vacuum state is determined and the transition to constituent quarks and gluons has been achieved. At this point we can apply, for example, a Tamm-Dancoff truncation and consider hadronic states built from a small number of constituents. This truncation can perhaps be justified by the emergence of constituent-scale masses for the effective degrees of freedom, which kinematically suppresses mixing with more complex, multi-constituent states. A similar truncation in the bare parton basis (current quarks and mass zero gluons) would be essentially meaningless.

Second, since the cut-off is defined in terms of free energy differences it is not fully Lorentz invariant (though it is rotationally invariant, of course). This is unavoidable in a Hamiltonian framework, particularly if positivity of the Hilbert space norm is to be preserved (thus ruling out, e.g., the Pauli-Villars scheme). Indeed we conjecture that requiring positivity forces one to employ a noncovariant gauge. The renormalized effective Hamiltonian will therefore contain Lorentz-noninvariant operators, that is, operators which correspond to Lorentz-noninvariant terms at the Lagrangian level. The regulator also violates gauge invariance, though this may be of less significance since we work in a fixed gauge. Nevertheless our renormalization procedure, to be detailed below, will automatically provide the counterterms necessary to restore both symmetries.

Finally, this scheme does not completely regulate the theory. Normal ordering the two-body operators in the canonical Hamiltonian leads to one-body operators [see Eqs. (A4) and (A8) in the Appendix] with divergent matrix elements that are not regulated by the above procedure. For these matrix elements we insert an additional cut-off factor, $\exp \left(-2 \omega_{k^{\prime}}^{2} / \Lambda^{2}\right)$, in the integrands of Eqs. (A4) and (A8). ${ }^{3}$ While there is substantial freedom in implementing this supplementary regularization, observables computed from the Hamiltonian will be independent of the cut-off prescription. We remark on this below.

At this point we have a fully regulated Hamiltonian formulation of QCD in the Coulomb gauge. The next task is to remove the dependence on the cut-off parameter $\Lambda$ by adding counterterms to the Hamiltonian. This will be done perturbatively, which should be reasonable for QCD if the cut-off is not too low. The resulting renormalized effective Hamiltonian, $H_{\text {eff }}(\Lambda)$, can then be analyzed nonperturbatively using many-body techniques.

\section{CUT-OFF DEPENDENCE AND RENORMALIZATION}

\section{A. Counterterm Hamiltonian and form invariance}

Our goal is to construct the renormalized effective Hamiltonian, $H_{\text {eff }}(\Lambda)$, which contains a cut-off but gives cut-off

\footnotetext{
${ }^{3}$ Of course, we could simply omit these operators from the Hamiltonian since our goal is to remove the cut-off dependence by adding counterterms. However, the divergences from these terms cancel divergences elsewhere in the theory so we retain them.
}

independent results through some fixed order in perturbation theory. It can be expressed as the sum of the canonical Hamiltonian and a "counterterm" Hamiltonian

$$
H_{\mathrm{eff}}(\Lambda)=H_{\mathrm{can}}(\Lambda)+H_{\mathrm{CT}}(\Lambda) .
$$

The canonical Hamiltonian contains normal ordering contributions with integrals regulated as previously described. The counterterm Hamiltonian, which begins at order $g^{2}$ in perturbation theory, has operators with the same structure along with additional terms necessary to correct for violations of Lorentz invariance introduced by the regulator. Note also that the matrix elements of $H_{\mathrm{CT}}$ depend explicitly on $\Lambda$ in addition to containing the regulating exponentials. In general,

$$
H_{\mathrm{CT}}(\Lambda)=\sum_{i} c_{i}(\Lambda) \Lambda^{n_{i}} \mathcal{O}_{i}^{\Lambda}
$$

where the $\left\{\mathcal{O}_{i}^{\Lambda}\right\}$ are a complete set of local operators invariant under the symmetries preserved by the regulator. The superscript $\Lambda$ indicates that the regulating functions are to be associated with the operators $\mathcal{O}_{i}$; that is,

$$
\left\langle n\left|\mathcal{O}_{i}^{\Lambda}\right| m\right\rangle \equiv e^{-E_{n m}^{2} / \Lambda^{2}}\left\langle n\left|\mathcal{O}_{i}\right| m\right\rangle
$$

where $\mathcal{O}_{i}$ is the "bare" operator. The explicit powers of $\Lambda$ are inserted in Eq. (4.2) so that the coefficients $c_{i}(\Lambda)$ are dimensionless $\left(n_{i}\right.$ is four minus the mass dimension of the operator $\mathcal{O}_{i}$ ). Operators for which $n_{i}<0$ are irrelevant in the renormalization group sense and will generally be ignored. Note that the coefficients $c_{i}$ can depend on $\Lambda$ only through their dependence on the "canonical'" couplings of the theory. Thus

$$
c_{i}(\Lambda)=\widetilde{c}_{i} g^{2}(\Lambda)+\cdots
$$

where the ellipsis indicates higher order terms in the perturbative expansion and for simplicity only a single independent coupling has been assumed. The pure numbers, $\widetilde{c}_{i}$, are independent of $\Lambda$. Thus the coefficients depend logarithmically on $\Lambda$ and one may only specify the change in the coefficient. This may be contrasted with, for example, a gluon mass counterterm which arises solely because the regulator violates gauge invariance. In this case the gluon mass is completely calculable within perturbation theory.

An exhaustive way of determining $H_{\mathrm{CT}}$ would be to list all permissible operators and then extract the $c_{i}$ by requiring observables calculated perturbatively be both cut-off independent and Lorentz covariant. A more appealing, physically equivalent, approach adopted here is to perform a similarity transformation on the Hamiltonian which reduces $\Lambda$ to a new cut-off $\Lambda_{1}$ in the regulating exponentials. Because the transformed Hamiltonian is equivalent to the original one, calculated observables (e.g., eigenvalues) are unchanged. In addition to replacing $\Lambda \rightarrow \Lambda_{1}$ in $H_{\text {can }}$, the transformation also generates new, effective interactions representing the physics between the scales $\Lambda$ and $\Lambda_{1}$. Schematically, we have

$$
H_{\mathrm{can}}(\Lambda) \rightarrow H_{\mathrm{can}}\left(\Lambda_{1}\right)+\delta H\left(\Lambda_{1}, \Lambda\right)
$$


under the similarity transformation. The counterterm Hamiltonian is then determined by requiring that the full, transformed Hamiltonian have the same form as the original one, but with $\Lambda$ everywhere replaced by $\Lambda_{1}$. This requirement of form invariance, or coherence, of the Hamiltonian under changes of the cut-off ensures that we have the most general Hamiltonian allowed $[8,11]$. In the language of the renormalization group (RG), the self-similar Hamiltonian exists on an infinitely long RG trajectory and thus constitutes a renormalized Hamiltonian.

We now sketch this procedure to first order in $\alpha_{s}$ $\equiv g^{2} / 4 \pi$. In this case $H_{\mathrm{CT}}$ is of order $\alpha_{s}$ and since the similarity transformation leaves $H_{\mathrm{CT}}$ unchanged to this order (apart from replacing $\Lambda$ by $\Lambda_{1}$ in just the regulating functions) we have

$$
H_{\text {eff }}(\Lambda) \rightarrow H_{\text {can }}\left(\Lambda_{1}\right)+\delta H\left(\Lambda_{1}, \Lambda\right)+H_{\mathrm{CT}}(\Lambda) .
$$

In this expression $H_{\mathrm{CT}}$ is understood to have $\Lambda$ replaced by $\Lambda_{1}$ in the regulating functions but nowhere else. We now demand that the transformed Hamiltonian be equal to

$$
H_{\mathrm{eff}}\left(\Lambda_{1}\right) \equiv H_{\mathrm{can}}\left(\Lambda_{1}\right)+H_{\mathrm{CT}}\left(\Lambda_{1}\right)
$$

This uniquely determines the structure of $H_{\mathrm{CT}}$.

A prescription for treating the cut-off dependent terms from normal ordering must also be specified since these are not regulated by the cut-off on energy differences and are also unaffected by the similarity transformation. Our prescription is that upon performing the similarity transformation, we replace $\Lambda$ by $\Lambda_{1}$ in these terms in the canonical Hamiltonian and include the difference between this and the original terms-the "slice" coming from momenta between $\Lambda_{1}$ and $\Lambda$-in $\delta H$.

We first determine contributions to $\delta H$ coming from the normal-ordering terms. The one-body operators in the effective Hamiltonian have the general form

$$
\begin{aligned}
H_{\mathrm{eff}}^{(2)}(\Lambda)= & \int \frac{d^{3} k}{(2 \pi)^{3}}\left\{\left[\omega_{k} \delta_{i j} \delta^{a b}+\Pi_{i j}^{a b}(\mathbf{k})\right] a_{i}^{a \dagger}(\mathbf{k}) a_{j}^{b}(\mathbf{k})\right. \\
& \left.+M_{i j}^{a b}(\mathbf{k}) e^{-\left(2 \omega_{k}\right)^{2} / \Lambda^{2}}\left[a_{i}^{a}(\mathbf{k}) a_{j}^{b}(-\mathbf{k})+\text { H.c. }\right]\right\}
\end{aligned}
$$

Note the presence of the regulating function in the second term. From rotational invariance,

$$
\Pi_{i j}^{a b}(\mathbf{k})=A^{a b}(k) \delta_{i j}+B^{a b}(k) k_{i} k_{j},
$$

with similar structure for $M_{i j}^{a b}$. Due to transversality, however, only the term proportional to $\delta_{i j}$ need be considered. From Eqs. (A4) and (A8) we can identify the contributions to $\Pi_{i j}^{a b}$ and $M_{i j}^{a b}$ arising from normal ordering the two-body operators. From the Coulomb term we have

$$
\begin{aligned}
\Pi_{i j}^{(C) a b}(k)= & \frac{\alpha_{s}}{4} N_{c} \delta^{a b} \int \frac{d^{3} k^{\prime}}{(2 \pi)^{3}} \widetilde{V}\left(\mathbf{k}+\mathbf{k}^{\prime}\right) \\
& \times\left(\frac{\omega_{k}^{2}+\omega_{k^{\prime}}^{2}}{\omega_{k} \omega_{k^{\prime}}}\right) D_{i j}\left(\mathbf{k}^{\prime}\right) e^{-2 \omega_{k^{\prime}}^{2}}
\end{aligned}
$$

and

$$
\begin{aligned}
M_{i j}^{(C) a b}(k)= & \frac{\alpha_{s}}{8} N_{c} \delta^{a b} \int \frac{d^{3} k^{\prime}}{(2 \pi)^{3}} \widetilde{V}\left(\mathbf{k}+\mathbf{k}^{\prime}\right) \\
& \times\left(\frac{\omega_{k^{\prime}}^{2}-\omega_{k}^{2}}{\omega_{k} \omega_{k^{\prime}}}\right) D_{i j}\left(\mathbf{k}^{\prime}\right) e^{-2 \omega_{k^{\prime}}^{2} / \Lambda^{2}} .
\end{aligned}
$$

Next the cut-off is reduced by an infinitesimal amount, $\Lambda^{2}$ $\rightarrow \Lambda_{1}^{2}$, with

$$
\Lambda_{1}^{2} \equiv(1-\epsilon) \Lambda^{2} ; \quad \epsilon>0 .
$$

The difference

$\delta \Pi_{i j}^{(C) a b}(k ; \Lambda, \epsilon) \equiv \Pi_{i j}^{(C) a b}(k ; \Lambda)-\Pi_{i j}^{(C) a b}\left(k ; \Lambda_{1}\right)$

to be included in $\delta H$ is then expanded to first order in $\epsilon$, followed by an expansion in powers of $k / \Lambda$. Retaining only those terms which correspond to relevant or marginal operators in the renormalization group sense yields ${ }^{4}$

$$
\delta \Pi_{i j}^{(C) a b}(k ; \Lambda, \epsilon)=\frac{\alpha_{s}}{4 \pi} N_{c} \delta_{i j} \delta^{a b}\left(\frac{\epsilon}{\omega_{k}}\right)\left[\frac{1}{3} \Lambda^{2}+\frac{16}{15} k^{2}+\cdots\right],
$$

where the dots represent terms of order $k / \Lambda$ and higher. Similarly we obtain

$$
\delta M_{i j}^{(C) a b}(k ; \Lambda, \epsilon)=\frac{\alpha_{s}}{4 \pi} N_{c} \delta_{i j} \delta^{a b}\left(\frac{\epsilon}{\omega_{k}}\right)\left[\frac{1}{6} \Lambda^{2}-\frac{2}{15} k^{2}+\cdots\right] .
$$

Performing the same steps for the divergent contributions in $H_{g}$ [Eq. (A4)] produces

$$
\delta \Pi_{i j}^{(g) a b}(k ; \Lambda, \epsilon)=\frac{\alpha_{s}}{4 \pi} N_{c} \delta_{i j} \delta^{a b}\left(\frac{\epsilon}{\omega_{k}}\right)\left(\frac{2}{3} \Lambda^{2}+\cdots\right)
$$

and

$$
\delta M_{i j}^{(g) a b}(k ; \Lambda, \epsilon)=\frac{\alpha_{s}}{4 \pi} N_{c} \delta_{i j} \delta^{a b}\left(\frac{\epsilon}{\omega_{k}}\right)\left(\frac{1}{3} \Lambda^{2}+\cdots\right) .
$$

\section{B. Similarity transformation}

We now develop the similarity transformation renormalization procedure. This follows the formulation of Wegner [12] who derived flow equations for diagonalizing a Hamil-

\footnotetext{
${ }^{4}$ It may be necessary to retain some irrelevant operators if the cut-off is reduced much below the scale of physical interest, but for the present we ignore these.
} 
tonian. Consider a continuous transformation governed by a unitary operator $U$ which depends upon a (flow) parameter $\lambda$. Let $T(\lambda)$ be the generator of the corresponding infinitesimal transformation. Then for a continuous transformation from $\lambda_{i}$ to $\lambda_{f}$

$$
U\left(\lambda_{f} ; \lambda_{i}\right)=e^{\int_{\lambda_{i}}^{\lambda_{f}} T(\lambda) d \lambda},
$$

where $\lambda$-ordering of the exponential is understood. Note that $T$ must be anti-Hermitian since $U^{\dagger}=U^{-1}$. The similarity transformation for a Hamiltonian which also depends on $\lambda$ is then given by

$$
H\left(\lambda_{f}\right)=U\left(\lambda_{f} ; \lambda_{i}\right) H\left(\lambda_{i}\right) U^{\dagger}\left(\lambda_{f} ; \lambda_{i}\right),
$$

and for an infinitesimal evolution

$$
\frac{d H(\lambda)}{d \lambda}=[T(\lambda), H(\lambda)]
$$

Wegner has determined that the generator choice

$$
T(\lambda)=\left[H_{0}, H(\lambda)\right]
$$

suppresses the off-diagonal matrix elements thereby rendering $H$ "'more diagonal" as $\lambda$ increases. We have independently obtained the same generator choice by requiring that the transformation properly change the cut-off parameter in the regulating functions for all matrix elements of our Hamiltonian. We now demonstrate this but first note, from Eqs. (4.20), (4.21), the dimensions of $\lambda$ are $\left[E^{-2}\right]$ so to work directly with our energy cut-off parameter we substitute $\lambda$ $\rightarrow \Lambda^{-2}$. Then combining the above equations yields

$$
\frac{d H(\Lambda)}{d \Lambda^{-2}}=\left[\left[H_{0}, H(\Lambda)\right], H(\Lambda)\right] .
$$

By expanding the interaction Hamiltonian in powers of the coupling at this scale $\Lambda$,

$$
H_{I}(\Lambda)=\sum_{p=1}^{\infty} h_{p}
$$

we can examine Eq. (4.22) order by order. At zeroth order

$$
\frac{d H_{0}}{d \Lambda^{-2}}=0
$$

so that the transformation preserves the cut-off independence of the free Hamiltonian. At first order we find

$$
\frac{d h_{1}(\Lambda)}{d \Lambda^{-2}}=\left[\left[H_{0}, h_{1}\right], H_{0}\right]
$$

Expressed in terms of matrix elements this becomes

$$
\frac{d}{d \Lambda^{-2}}\left\langle n\left|h_{1}(\Lambda)\right| m\right\rangle=-E_{n m}^{2}\left\langle n\left|h_{1}(\Lambda)\right| m\right\rangle
$$

Integrating Eq. (4.26) from $\Lambda$ to $\Lambda_{1}$ then yields $\left\langle n\left|h_{1}\left(\Lambda_{1}\right)\right| m\right\rangle=e^{-E_{n m}^{2}\left(1 / \Lambda_{1}^{2}-1 / \Lambda^{2}\right)}\left\langle n\left|h_{1}(\Lambda)\right| m\right\rangle$.

Since

$$
\left\langle n\left|h_{1}(\Lambda)\right| m\right\rangle \propto e^{-E_{n m}^{2} / \Lambda^{2}}
$$

we see the the transformation has indeed replaced $\Lambda \rightarrow \Lambda_{1}$ in the regulating exponentials for these terms.

The expression at second order reads

$$
\begin{aligned}
\frac{d}{d \Lambda^{-2}} & \left\langle n\left|h_{2}(\Lambda)\right| m\right\rangle \\
= & -E_{n m}^{2}\left\langle n\left|h_{2}(\Lambda)\right| m\right\rangle+\sum_{l}\left(E_{n}+E_{m}-2 E_{l}\right) \\
& \times\left\langle n\left|h_{1}(\Lambda)\right| l\right\rangle\left\langle l\left|h_{1}(\Lambda)\right| m\right\rangle .
\end{aligned}
$$

Once again the first term on the right-hand side has the effect of replacing $\Lambda \rightarrow \Lambda_{1}$ in the overall regulating exponential. It is trivial to see that this same structure occurs for each order, so that the transformation does indeed replace $\Lambda \rightarrow \Lambda_{1}$ in all matrix elements of the Hamiltonian. It is convenient to isolate this explicit cut-off dependence by defining "reduced" matrix elements, denoted by $V_{p}$ :

$$
\left\langle n\left|h_{p}(\Lambda)\right| m\right\rangle \equiv e^{-E_{n m}^{2} / \Lambda^{2}}\left\langle n\left|V_{p}(\Lambda)\right| m\right\rangle .
$$

Notice that $V_{1}$ does not depend on $\Lambda$. In terms of $V_{2}$, Eq. (4.29) reads

$$
\begin{aligned}
& e^{-E_{n m}^{2} / \Lambda^{2}} \frac{d}{d \Lambda^{-2}}\left\langle n\left|V_{2}(\Lambda)\right| m\right\rangle \\
& \quad=\sum_{l}\left(E_{n l}+E_{m l}\right) e^{-\left(E_{n l}^{2}+E_{m l}^{2}\right) / \Lambda^{2}}\left\langle n\left|V_{1}\right| l\right\rangle\left\langle l\left|V_{1}\right| m\right\rangle .
\end{aligned}
$$

This can be integrated to give

$$
\left\langle n\left|\delta V_{2}\right| m\right\rangle=\sum_{l} \Theta\left(E_{n l}, E_{m l}\right)\left\langle n\left|V_{1}(\Lambda)\right| l\right\rangle\left\langle l\left|V_{1}(\Lambda)\right| m\right\rangle,
$$

where

$$
\left\langle n\left|\delta V_{2}\right| m\right\rangle \equiv\left\langle n\left|V_{2}\left(\Lambda_{1}\right)\right| m\right\rangle-\left\langle n\left|V_{2}(\Lambda)\right| m\right\rangle
$$

This change in the interaction explicitly represents the physics removed in reducing the cut-off from $\Lambda$ to $\Lambda_{1}$, to this order in perturbation theory. The quantity

$$
\Theta\left(E_{n l}, E_{m l}\right) \equiv \frac{1}{2}\left(\frac{1}{E_{n l}}+\frac{1}{E_{m l}}\right)\left(e^{-2 E_{n l} E_{m l} / \Lambda^{2}}-e^{-2 E_{n l} E_{m l} / \Lambda_{1}^{2}}\right)
$$

plays the role of an energy denominator in conventional perturbation theory. Note that cancellation between the two exponentials suppresses the contribution when the energy differences are small. Thus the potentially singular factors 
$1 / E_{n l}$, etc., are not problematic. It is straightforward to extend this procedure to higher orders.

We can now compute the various one-body operators induced by the similarity transformation. Consider first the contribution to $\delta \Pi$ arising from $q \bar{q}$ intermediate states. It is given by

$$
\begin{aligned}
\delta \Pi_{i j}^{(q u a r k, 1) a b}(k)= & \frac{g^{2} \delta^{a b}}{4 \omega_{k}} \sum_{s, s^{\prime}} \int \frac{d^{3} q}{(2 \pi)^{3}} \Theta(\Delta, \Delta) \\
& \times\left[u^{\dagger}(\mathbf{k}+\mathbf{q}, s) \alpha_{i} v\left(-\mathbf{q}, s^{\prime}\right)\right] \\
& \times\left[v^{\dagger}\left(-\mathbf{q}, s^{\prime}\right) \alpha_{j} u(\mathbf{k}+\mathbf{q}, s)\right],
\end{aligned}
$$

where

$$
\Delta \equiv \omega_{k}-E_{q}-E_{k+q} .
$$

We again expand this for a small change in the cut-off and discard contributions corresponding to irrelevant operators. The result is

$$
\begin{aligned}
\delta \Pi_{i j}^{(q u a r k, 1) a b}(k ; \Lambda, \epsilon)= & -\frac{\alpha_{s}}{4 \pi} N_{f} \delta_{i j} \delta^{a b}\left(\frac{\epsilon}{\omega_{k}}\right) \\
& \times\left(\frac{1}{12} \Lambda^{2}+\frac{1}{6} \sqrt{\frac{\pi}{2}} k \Lambda+\cdots\right),
\end{aligned}
$$

where the dots represent terms of order $1 / \Lambda$ and $N_{f}$ is the number of light (i.e., lighter than the cut-off scale) quark flavors. There is also a contribution arising from $q \bar{q} g g$ intermediate states (analogous to a "Z-graph" contribution) which gives

$$
\begin{aligned}
\delta \Pi_{i j}^{(q u a r k, 2) a b}(k ; \Lambda, \epsilon)= & -\frac{\alpha_{s}}{4 \pi} N_{f} \delta_{i j} \delta^{a b}\left(\frac{\epsilon}{\omega_{k}}\right) \\
& \times\left(\frac{1}{12} \Lambda^{2}-\frac{1}{6} \sqrt{\frac{\pi}{2}} k \Lambda+\cdots\right) .
\end{aligned}
$$

The complete contribution arising from multi-quark intermediate states is thus

$$
\delta \Pi_{i j}^{(\text {quark }) a b}(k ; \Lambda, \epsilon)=-\frac{\alpha_{s}}{4 \pi} N_{f} \delta_{i j} \delta^{a b}\left(\frac{\epsilon}{\omega_{k}}\right)\left(\frac{1}{6} \Lambda^{2}+\cdots\right) .
$$

The contribution from multi-gluon intermediate states may be evaluated similarly; the result is

$$
\begin{aligned}
\delta \Pi_{i j}^{(g l u e) a b}(k ; \Lambda, \epsilon)= & -\frac{\alpha_{s}}{4 \pi} N_{c} \delta_{i j} \delta^{a b}\left(\frac{\epsilon}{\omega_{k}}\right) \\
& \times\left(\frac{1}{12} \Lambda^{2}+\frac{16}{15} k^{2}+\cdots\right) .
\end{aligned}
$$

Combining these results with the contributions from the normal-ordering terms gives the complete $\delta \Pi$ at this order:

$$
\delta \Pi_{i j}^{a b}(k ; \Lambda, \epsilon)=\frac{\alpha_{s}}{16 \pi} \delta_{i j} \delta^{a b}\left(\frac{\epsilon}{\omega_{k}}\right)\left[\left(11-\frac{2 N_{f}}{3}\right) \Lambda^{2}+\cdots\right],
$$

where we have set $N_{c}=3$. We recognize the lowest-order coefficient of the QCD beta function $\beta_{0} \equiv 11-2 N_{f} / 3 .^{5}$

Next we consider the various contributions to $\delta M_{i j}^{a b}$. The $q \bar{q} g$ intermediate states yield

$$
\delta M_{i j}^{(\text {quark }) a b}(k ; \Lambda, \epsilon)=-\frac{\alpha_{s}}{4 \pi} N_{f} \delta_{i j} \delta^{a b}\left(\frac{\epsilon}{\omega_{k}}\right)\left(\frac{1}{12} \Lambda^{2}+\cdots\right),
$$

while three-gluon intermediate states give

$$
\begin{aligned}
\delta M_{i j}^{(\text {glue }) a b}(k ; \Lambda, \epsilon)= & -\frac{\alpha_{s}}{4 \pi} N_{c} \delta_{i j} \delta^{a b}\left(\frac{\epsilon}{\omega_{k}}\right) \\
& \times\left(\frac{1}{24} \Lambda^{2}+\frac{8}{15} k^{2}+\cdots\right) .
\end{aligned}
$$

Combining these results with the contributions from the normal-ordering terms gives

$$
\delta M_{i j}^{a b}(k ; \Lambda, \epsilon)=\frac{\alpha_{s}}{16 \pi} \delta_{i j} \delta^{a b}\left(\frac{\epsilon}{\omega_{k}}\right)\left[\frac{1}{2} \beta_{0} \Lambda^{2}-8 k^{2}+\cdots\right]
$$

for $N_{c}=3$.

One-body operators in the counterterm Hamiltonian have the general form

$$
\begin{aligned}
H_{\mathrm{CT}}(\Lambda)= & \int \frac{d^{3} k}{(2 \pi)^{3}}\left(\Pi_{\mathrm{CT}}(k ; \Lambda) a_{i}^{a \dagger}(\mathbf{k}) a_{i}^{a}(\mathbf{k})\right. \\
& \left.+M_{\mathrm{CT}}(k ; \Lambda) e^{-\left(2 \omega_{k}\right)^{2} / \Lambda^{2}}\left[a_{i}^{a}(\mathbf{k}) a_{i}^{a}(-\mathbf{k})+\text { H.c. }\right]\right),
\end{aligned}
$$

since the cut-off dependent terms are all proportional to $\delta_{i j} \delta^{a b}$. Let us focus first on the term in $\Pi_{\mathrm{CT}}$ that depends quadratically on $\Lambda$. On dimensional grounds one has

$$
\Pi_{\mathrm{CT}}=\frac{\alpha_{s}}{4 \pi}\left(\frac{1}{2 \omega_{k}}\right) b \Lambda^{2}+\cdots,
$$

where $b$ is a numerical constant independent of $\Lambda$. After the similarity transformation we have

$$
H_{\mathrm{can}}(\Lambda)+H_{\mathrm{CT}}(\Lambda) \rightarrow H_{\mathrm{can}}\left(\Lambda_{1}\right)+\delta H\left(\Lambda_{1}, \Lambda\right)+H_{\mathrm{CT}}(\Lambda),
$$

where $\delta H$ represents the contributions computed above. Note that to this order, the only change to $H_{\text {can }}(\Lambda)$ is to replace $\Lambda$ with $\Lambda_{1}$. Equating the second and third terms on the right-hand side of Eq. (4.47) to $H_{\mathrm{CT}}\left(\Lambda_{1}\right)$ yields, using Eq. (4.46),

\footnotetext{
${ }^{5}$ This is purely accidental since an alternative regulator choice in the normal-ordering terms gives a different result.
} 


$$
\begin{aligned}
\frac{1}{2} \epsilon \beta_{0} \Lambda^{2}+b \Lambda^{2} & =b \Lambda_{1}^{2} \\
& =b \Lambda^{2}(1-\epsilon) .
\end{aligned}
$$

Thus $b=-\beta_{0} / 2$, and

$$
H_{\mathrm{CT}}(\Lambda)=-\frac{\alpha_{s} \beta_{0} \Lambda^{2}}{8 \pi} \int \frac{d^{3} k}{(2 \pi)^{3}}\left(\frac{1}{2 \omega_{k}}\right) a_{i}^{a \dagger}(\mathbf{k}) a_{i}^{a}(\mathbf{k})+\cdots
$$

It may be verified that this counterterm removes the quadratically divergent part of the gluon self energy to second order, as it should. Of course, this is simply (part of) a gluon mass term with the mass given by

$$
m_{g}^{2}=-\frac{\alpha_{s} \beta_{0} \Lambda^{2}}{8 \pi}
$$

Note that the quadratically divergent part of $\delta M$ has exactly this structure. Therefore, when expressed in terms of the field operators the complete quadratically divergent part of the counterterm Hamiltonian is simply

$$
H_{\mathrm{CT}}(\Lambda)=\frac{m_{g}^{2}}{2} \int d^{3} x A_{i}^{a}(\mathbf{x}) A_{i}^{a}(\mathbf{x})+\cdots
$$

The remaining operators in $H_{\mathrm{CT}}$ may be similarly constructed. Dimensionally, the term in Eq. (4.44) containing the factor $-8 k^{2}$ represents a logarithmic divergence. Hence, it should correspond to the only logarithmic divergence to which we are sensitive at this order, namely the gluon wave function renormalization. To see this we invert the Fourier expansions and express this operator in terms of the fields to obtain

$$
\delta H=-\epsilon \frac{\alpha_{s}}{4 \pi}\left(\frac{4 C_{A}}{3}\right) \operatorname{Tr} \int d^{3} x\left(\mathbf{B}^{2}-\mathbf{\Pi}^{2}\right),
$$

where we have restored the dependence on the number of colors $\left[C_{A}=N_{c}\right.$ is the Casimir invariant of the adjoint representation of $\left.\mathrm{SU}\left(N_{c}\right)\right]$. This has precisely the correct structure for a wave function renormalization since, if the gauge field is rescaled by $A \rightarrow Z_{A}^{1 / 2} A$ then the conjugate momentum is rescaled by the inverse factor, $\Pi \rightarrow Z_{A}^{-1 / 2} \Pi{ }^{6}$

The counterterm Hamiltonian will thus contain a term

$$
H_{\mathrm{CT}}=\left.c(\Lambda) \operatorname{Tr} \int d^{3} x\left(\mathbf{B}^{2}-\mathbf{\Pi}^{2}\right)\right|_{g=0}
$$

\footnotetext{
${ }^{6} \mathrm{~A}$ quick way to see this is to note that in a functional Schrödinger representation $\Pi=-i \delta / \delta A$.
}

at this order, with $c(\Lambda)$ logarithmically dependent on $\Lambda$. The requirement of coherence of the Hamiltonian under a change of the cut-off from $\Lambda \rightarrow \Lambda_{1}$ then gives

$$
-\epsilon \frac{\alpha_{s}}{4 \pi}\left(\frac{4 C_{A}}{3}\right)+c(\Lambda)=c\left(\Lambda_{1}\right) .
$$

Expanding $c\left(\Lambda_{1}\right)$ about $\Lambda_{1}=\Lambda$ yields

$$
\Lambda \frac{d}{d \Lambda} c(\Lambda)=\frac{\alpha_{s}}{4 \pi}\left(\frac{8 C_{A}}{3}\right)
$$

to $\mathcal{O}(\epsilon)$ and hence

$$
c(\Lambda)=\frac{\alpha_{s}}{4 \pi}\left(\frac{8 C_{A}}{3}\right) \ln \left(\Lambda / \Lambda_{0}\right)
$$

where $\Lambda_{0}$ is an arbitrary scale.

As discussed above, one can interpret this counterterm as corresponding to a renormalization of the gauge field with form

$$
A^{\prime}=\left[1+\frac{1}{2} c(\Lambda)\right] A \equiv Z_{A}^{1 / 2} A .
$$

Thus, the free part of $H_{\text {can }}$ combines with this part of the counterterm Hamiltonian to simply produce a free Hamiltonian when written in terms of $A^{\prime}$. The gauge field anomalous dimension is now easily computed to be

$$
\begin{aligned}
\gamma_{A} & =-\frac{1}{2} \Lambda \frac{d}{d \Lambda} \ln Z_{A} \\
& =-\frac{g^{2}}{16 \pi^{2}}\left(\frac{4 C_{A}}{3}\right) .
\end{aligned}
$$

At lowest order, there is no contribution to $\gamma_{A}$ from fermions in this scheme.

It is significant to note that with a sharp cut-off, the above results are again obtained, but with two differences. One is a slight difference in the value of the gluon mass. The other, which is problematic, is a contribution

$$
H_{N L}=\left(\frac{\alpha_{s}}{4 \pi}\right) \epsilon \beta_{0} \Lambda \int \frac{d^{3} k}{(2 \pi)^{3}}\left[a_{i}(\mathbf{k}) a_{i}(-\mathbf{k})+a_{i}^{\dagger}(\mathbf{k}) a_{i}^{\dagger}(-\mathbf{k})\right]
$$

to the counterterm Hamiltonian. This corresponds to a nonlocal interaction, as may easily be seen by inverting the field expansions and writing Eq. (4.59) in terms of the field operators

$$
\begin{aligned}
H_{N L}= & -\left(\frac{\alpha_{s}}{4 \pi}\right) \epsilon \beta_{0} \Lambda \int d^{3} x d^{3} y \\
& \times \frac{1}{|\mathbf{x}-\mathbf{y}|^{2}}\left[\mathbf{A}(\mathbf{x}) \cdot \nabla^{2} \mathbf{A}(\mathbf{y})+\Pi(\mathbf{x}) \cdot \mathbf{\Pi}(\mathbf{y})\right]
\end{aligned}
$$


The nonlocality arises because the interaction kernel is now a polynomial in $|\mathbf{k}|$ rather than $\mathbf{k}$.

\section{CONCLUSIONS}

The main, new result of this study was the rigorous derivation of a renormalized effective Hamiltonian to order $g^{2}$ in the strong coupling constant. This was achieved through a similarity transformation in conjunction with a continuous cut-off regularization scheme. The use of a sharp cut-off regularization led to an unacceptable non-local Hamiltonian. The effective Hamiltonian was determined by introducing an appropriate counterterm Hamiltonian and imposing form invariance on the transformed Hamiltonian. Renormalization not only restored the proper symmetries but also rendered all observables calculated with this Hamiltonian insensitive to the cut-off parameter.

Future formal work will address both the issue of confinement as well as renormalization in the combined quark-gluon sector. In particular both perturbative and non-perturbative approaches will be examined. Such a treatment is fundamental to understanding the role of gluons in the structure of mesons and baryons. Finally, utilizing many-body techniques, large scale applications of the effective Hamiltonian focusing upon glueball and hybrid meson systems will be reported.

\section{ACKNOWLEDGMENTS}

It is a pleasure to thank B. Allen, G. P. Lepage and D. Zwanziger for several informative discussions. This work was supported by DOE grants DE-FG02-96ER40944, DEFG02-97ER41048 and DE-FG02-96ER40947.

\section{APPENDIX: CANONICAL HAMILTONIAN}

In this Appendix we present the canonical Hamiltonian expressed in the Fock representation. The free Hamiltonian is simply

$$
\begin{aligned}
H_{0}= & \int \frac{d^{3} k}{(2 \pi)^{3}} \omega_{k} a_{i}^{a^{\dagger}}(\mathbf{k}) a_{i}^{a}(\mathbf{k}) \\
& +\sum_{s} \int \frac{d^{3} k}{(2 \pi)^{3}} E_{k}\left[b^{\dagger}(\mathbf{k}, s) b(\mathbf{k}, s)+d^{\dagger}(\mathbf{k}, s) d(\mathbf{k}, s)\right],
\end{aligned}
$$

where $E_{k}=\sqrt{\mathbf{k}^{2}+m^{2}}$ and $\omega_{k}=|\mathbf{k}|$. The interaction terms will be separated according to their term of origin in Eq. (2.1). From the purely gluonic term, $H_{g}$, we have three-point couplings to $\mathcal{O}(g)$

$$
\begin{aligned}
H_{g}^{(3)}= & \frac{i g}{2 \sqrt{2}} f^{a b c} \int\left(\prod_{n=1}^{3} \frac{d^{3} k_{n}}{(2 \pi)^{3}}\right) \frac{k_{1 j}}{\sqrt{\omega_{1} \omega_{2} \omega_{3}}}(2 \pi)^{3} \\
& \times \delta^{(3)}\left(\sum_{m} \mathbf{k}_{m}\right):\left[a_{i}^{a}\left(\mathbf{k}_{1}\right)+a_{i}^{a \dagger}\left(-\mathbf{k}_{1}\right)\right] \\
& \times\left[a_{j}^{b}\left(\mathbf{k}_{2}\right)+a_{j}^{b^{\dagger}}\left(-\mathbf{k}_{2}\right)\right]\left[a_{i}^{c}\left(\mathbf{k}_{3}\right)+a_{i}^{c \dagger}\left(-\mathbf{k}_{3}\right)\right]:,
\end{aligned}
$$

where $\omega_{1} \equiv \omega_{\mathbf{k}_{1}}$, etc.

For $\mathcal{O}\left(g^{2}\right)$ we obtain (normal-ordered) four-point couplings

$$
\begin{aligned}
H_{g}^{(4)}= & \frac{\alpha_{s} \pi}{4} f^{a b c} f^{a d e} \int\left(\prod_{n=1}^{4} \frac{d^{3} k_{n}}{(2 \pi)^{3}}\right) \frac{1}{\sqrt{\omega_{1} \omega_{2} \omega_{3} \omega_{4}}}(2 \pi)^{3} \\
& \times \delta^{(3)}\left(\sum_{m} \mathbf{k}_{m}\right):\left[a_{i}^{b}\left(\mathbf{k}_{1}\right)+a_{i}^{b^{\dagger}}\left(-\mathbf{k}_{1}\right)\right] \\
& \times\left[a_{j}^{c}\left(\mathbf{k}_{2}\right)+a_{j}^{c^{\dagger}}\left(-\mathbf{k}_{2}\right)\right]\left[a_{i}^{d}\left(\mathbf{k}_{3}\right)+a_{i}^{d \dagger}\left(-\mathbf{k}_{3}\right)\right] \\
& \times\left[a_{j}^{e}\left(\mathbf{k}_{4}\right)+a_{j}^{e^{\dagger}}\left(-\mathbf{k}_{4}\right)\right]:
\end{aligned}
$$

where $\alpha_{s} \equiv g^{2} / 4 \pi$. In addition, there are one-body operators which arise in normal ordering Eq. (A3):

$$
\begin{aligned}
H_{g}^{(2)}= & \alpha_{s} \pi N_{c} \int \frac{d^{3} k d^{3} k^{\prime}}{(2 \pi)^{6}} \frac{1}{\omega_{k} \omega_{k^{\prime}}}\left[2 \delta_{i j}-D_{i j}\left(\mathbf{k}^{\prime}\right)\right] e^{-2 \omega_{k^{\prime}}^{2} / \Lambda^{2}} \\
& \times\left[a_{i}^{a \dagger}(\mathbf{k}) a_{j}^{a}(\mathbf{k})+\frac{1}{2}\left(a_{i}^{a}(\mathbf{k}) a_{j}^{a}(-\mathbf{k})+\text { H.c. }\right)\right],
\end{aligned}
$$

where $N_{c}$ is the number of colors and a quadratic divergence has been regulated by introducing an additional damping exponential.

The Coulomb term, Eq. (2.4), has a similar structure through $\mathcal{O}\left(g^{2}\right)$. To this order we may set $\nabla \cdot \mathbf{D}=\nabla^{2}$, so that

$$
H_{C}=\frac{\alpha_{s}}{2} \int d^{3} x d^{3} y \frac{\rho^{a}(\mathbf{x}) \rho^{a}(\mathbf{y})}{|\mathbf{x}-\mathbf{y}|}+\mathcal{O}\left(g^{3}\right) .
$$

Writing this in normal order generates two- and one-body operators. We shall exhibit here only those terms involving the gluonic component of the color charge density; the purely fermionic contributions may be found in Ref. [3].

The two-body operators involving only gluons are

$$
\begin{aligned}
H_{C}^{(4, g g)}= & -\frac{\alpha_{s}}{8} f^{a b c} f^{a d e} \int\left(\prod_{n=1}^{4} \frac{d^{3} k_{n}}{(2 \pi)^{3}}\right)\left(\frac{\omega_{2} \omega_{4}}{\omega_{1} \omega_{3}}\right)^{1 / 2} \\
& \times \widetilde{V}\left(\mathbf{k}_{1}+\mathbf{k}_{2}\right)(2 \pi)^{3} \delta^{(3)}\left(\sum_{m} \mathbf{k}_{m}\right) \\
& \times:\left[a_{i}^{b}\left(\mathbf{k}_{1}\right)+a_{i}^{b^{\dagger}}\left(-\mathbf{k}_{1}\right)\right]\left[a_{i}^{c}\left(\mathbf{k}_{2}\right)-a_{i}^{c^{\dagger}}\left(-\mathbf{k}_{2}\right)\right] \\
& \times\left[a_{j}^{d}\left(\mathbf{k}_{3}\right)+a_{j}^{d^{\dagger}}\left(-\mathbf{k}_{3}\right)\right]\left[a_{j}^{e}\left(\mathbf{k}_{4}\right)-a_{j}^{e^{\dagger}}\left(-\mathbf{k}_{4}\right)\right]:,
\end{aligned}
$$

where

$$
\widetilde{V}(\mathbf{k}) \equiv \frac{4 \pi}{k^{2}}
$$

is the momentum-space Coulomb potential. The one-body operators obtained in normal ordering Eq. (A6) are 


$$
\begin{aligned}
H_{C}^{(2)}= & \frac{\alpha_{s} N_{c}}{4} \int \frac{d^{3} k d^{3} k^{\prime}}{(2 \pi)^{6}} \widetilde{V}\left(\mathbf{k}+\mathbf{k}^{\prime}\right)\left(\frac{\omega_{k}^{2}+\omega_{k^{\prime}}^{2}}{\omega_{k} \omega_{k^{\prime}}}\right) D_{i j}\left(\mathbf{k}^{\prime}\right) e^{-2 \omega_{k^{\prime}}^{2} / \Lambda^{2}}\left[a_{i}^{a \dagger}(\mathbf{k}) a_{j}^{a}(\mathbf{k})\right] \\
& +\frac{\alpha_{s} N_{c}}{8} \int \frac{d^{3} k d^{3} k^{\prime}}{(2 \pi)^{6}} \widetilde{V}\left(\mathbf{k}+\mathbf{k}^{\prime}\right)\left(\frac{\omega_{k^{\prime}}^{2}-\omega_{k}^{2}}{\omega_{k} \omega_{k^{\prime}}}\right) D_{i j}\left(\mathbf{k}^{\prime}\right) e^{-2 \omega_{k^{\prime}}^{2} / \Lambda^{2}}\left[a_{i}^{a}(\mathbf{k}) a_{j}^{a}(\mathbf{k})+\text { H.c. }\right] .
\end{aligned}
$$

Again, quadratic divergences have been regulated with exponential cut-offs.

There are also mixed fermion-gluon two-body operators, given by

$$
\begin{aligned}
H_{C}^{(4, q g)}= & -\frac{i \alpha_{s}}{2} \sum_{s, s^{\prime}} \int \frac{d^{3} k d^{3} k^{\prime}}{(2 \pi)^{6}} \frac{d^{3} q d^{3} q^{\prime}}{(2 \pi)^{6}} f^{a b c} T_{\alpha \beta}^{a}\left(\frac{\omega_{\mathbf{q}^{\prime}}}{\omega_{\mathbf{q}}}\right)^{1 / 2} \widetilde{V}\left(\mathbf{q}+\mathbf{q}^{\prime}\right)(2 \pi)^{3} \delta^{(3)}\left(-\mathbf{k}+\mathbf{k}^{\prime}+\mathbf{q}+\mathbf{q}^{\prime}\right):\left[a_{i}^{b}(\mathbf{q})+a_{i}^{b \dagger}(-\mathbf{q})\right] \\
& \times\left[a_{i}^{c}\left(\mathbf{q}^{\prime}\right)-a_{i}^{c \dagger}\left(-\mathbf{q}^{\prime}\right)\right]\left(\left[u^{\dagger}(\mathbf{k}, s) u\left(\mathbf{k}^{\prime}, s^{\prime}\right)\right] b_{\alpha}^{\dagger}(\mathbf{k}, s) b_{\beta}\left(\mathbf{k}^{\prime}, s^{\prime}\right)+\left[v^{\dagger}(-\mathbf{k}, s) v\left(-\mathbf{k}^{\prime}, s^{\prime}\right)\right] d_{\alpha}(-\mathbf{k}, s) d_{\beta}^{\dagger}\left(-\mathbf{k}^{\prime}, s^{\prime}\right)\right. \\
& \left.+\left[u^{\dagger}(\mathbf{k}, s) v\left(-\mathbf{k}^{\prime}, s^{\prime}\right)\right] b_{\alpha}^{\dagger}(\mathbf{k}, s) d_{\beta}^{\dagger}\left(-\mathbf{k}^{\prime}, s^{\prime}\right)+\left[v^{\dagger}(-\mathbf{k}, s) u\left(\mathbf{k}^{\prime}, s^{\prime}\right)\right] d_{\alpha}(-\mathbf{k}, s) b_{\beta}\left(\mathbf{k}^{\prime}, s^{\prime}\right)\right):
\end{aligned}
$$

No tadpoles arise from normal ordering this operator since $T^{a}$ has zero trace and the $f^{a b c}$ are antisymmetric.

Finally there is the quark-gluon coupling term $H_{q g}$ [Eq. (2.5)]

$$
\begin{aligned}
H_{q g}= & -g \sum_{s, s^{\prime}} \int \frac{d^{3} k d^{3} k^{\prime} d^{3} q}{(2 \pi)^{9}} \frac{T_{\alpha \beta}^{a}}{\sqrt{2 \omega_{q}}}(2 \pi)^{3} \delta^{(3)}\left(-\mathbf{k}+\mathbf{k}^{\prime}+\mathbf{q}\right):\left[a_{i}^{a}(\mathbf{q})+a_{i}^{a \dagger}(-\mathbf{q})\right]\left(\left[u^{\dagger}(\mathbf{k}, s) \alpha_{i} u\left(\mathbf{k}^{\prime}, s^{\prime}\right)\right] b_{\alpha}^{\dagger}(\mathbf{k}, s) b_{\beta}\left(\mathbf{k}^{\prime}, s^{\prime}\right)\right. \\
& +\left[v^{\dagger}(-\mathbf{k}, s) \alpha_{i} v\left(-\mathbf{k}^{\prime}, s^{\prime}\right)\right] d_{\alpha}(-\mathbf{k}, s) d_{\beta}^{\dagger}\left(-\mathbf{k}^{\prime}, s^{\prime}\right)+\left[u^{\dagger}(\mathbf{k}, s) \alpha_{i} v\left(-\mathbf{k}^{\prime}, s^{\prime}\right)\right] b_{\alpha}^{\dagger}(\mathbf{k}, s) d_{\beta}^{\dagger}\left(-\mathbf{k}^{\prime}, s^{\prime}\right) \\
& \left.+\left[v^{\dagger}(-\mathbf{k}, s) \alpha_{i} u\left(\mathbf{k}^{\prime}, s^{\prime}\right)\right] d_{\alpha}(-\mathbf{k}, s) b_{\beta}\left(\mathbf{k}^{\prime}, s^{\prime}\right)\right):
\end{aligned}
$$

[1] F. J. Dyson, Phys. Rev. 75, 486 (1949); 75, 1736 (1949); 85, 631 (1952).

[2] A. Szczepaniak, E. S. Swanson, C.-R. Ji, and S. R. Cotanch, Phys. Rev. Lett. 76, 2011 (1996).

[3] A. Szczepaniak and E. S. Swanson, Phys. Rev. D 55, 1578 (1997).

[4] D. Zwanziger, Nucl. Phys. B485, 185 (1997); Report No. NYU-TH PH97205.

[5] V. N. Gribov, Nucl. Phys. B139, 1 (1978).

[6] Note that area law confinement has been claimed in the Abelian projection; see K. Kondo, Phys. Rev. D 58, 105016
(1998).

[7] St. D. Głazek and K. G. Wilson, Phys. Rev. D 48, 5863 (1993); 49, 4214 (1994).

[8] R. J. Perry, Ann. Phys. (N.Y.) 232, 116 (1994).

[9] A. Le Yaouanc, L. Oliver, O. Pène, and J.-C. Raynal, Phys. Rev. D 29, 1233 (1984); A. Le Yaouanc, L. Oliver, S. Ono, O. Pène, and J.-C. Raynal, ibid. 31, 137 (1985).

[10] T. D. Lee, Particle Physics and Introduction to Field Theory (Harwood Academic, Chur, Switzerland, 1982).

[11] R. J. Perry and K. G. Wilson, Nucl. Phys. B403, 587 (1993).

[12] F. Wegner, Ann. Phys. (Leipzig) 3, 77 (1994). 